\title{
The Mediating Role of Message Concreteness and Perceived Persuasiveness in Explaining the Match Effect Between Temporal Frame and Self-View
}

\author{
Seungae Lee and Kate Pounders
}

\begin{abstract}
Different temporal frames are often used in health communication to convey persuasive messages. This work investigates how temporal frames can be used more effectively depending on an individual's accessible self-view. This study found that a distal temporal frame is more effective with an independent self-view whereas a proximal temporal frame is more effective with an interdependent selfview. In addition, this chapter examines two mediating variables as being important in understanding the relationship between temporal frame and self-construal on behavioral intentions: persuasiveness and message concreteness. This study revealed that the cognitive information processing of the match effect varied depending on how concretely one construes health-risk-related information. This chapter adds to the existing literature by explaining the underlying mechanisms of the match effects between temporal frame and self-view.
\end{abstract}

S. Lee $(\bowtie) \cdot K$. Pounders

University of Texas at Austin, Austin, TX 78712, USA

e-mail: seungae.lee@utexas.edu; kate.pounders@austin.utexas.edu 